CASOS CLINICOS

Rev. Chil. Pedlatr. 65 (2); 114-116, 1994

\title{
Síndrome de Aicardi
}

\author{
Isabel López S. ${ }^{\text {; }}$ Fernando Pinto L. ${ }^{1,2}$; Cristián Luco F.4; Femando Novoa S. ${ }^{1,2}$
}

\begin{abstract}
Aicardi's syndrome
Two female infants with early seizures rapidly evolving to intantile spasms, sollosal agenesis and corioretinopathy wilh lacunar atrophy are reported. Both patlents had severe psychomotor retardation and vertebrol maltormations. These findings led lo the diagnosis of Aicordi's synd ome, an X-linked, dominanly inherited condition, that may be caused by a "de noro" mulation. As isolated major criteria for the diagnosis of tis syndrome may be also find in olher generic, metabolic diseases and congenilal infecctions, a careful differential diagnosis is needed including pertinent laboralory screening methods.
\end{abstract}

|Key words: Aicardi's syndrome, spasms, infontile, corpus callosum, agenesis, choriorellnitis.|

El síndrome de Aicardi ${ }^{1,2}$ consiste en la asociación de tres características fundamentales: espasmos infantiles, agenesia parcial o totaI del cuerpo calloso y anomalías del fondo de ojo descritas como lagunas coriorretineanas. Otras características son el retardo mental profundo en casi todas tas pacientes y alteraciones esqueléticas costovertebrales en dos tercjos de los casos informados. La identificación del síndrome de Aicardi requiere de un cuidadoso diagnóstico diferencial a partir de cada una de las características que to definen. Desde su descripción, en 1965, se han publicado más de 180 casos en el mundo, casi todos en niffas y raramente en personas con cariotipo $\mathrm{XXY}^{3}$. No se conoce la frecuencia de esta afección, y se la ha cncontrado en diversos grupos étnicos. Hasta donde llega nuestro conocimiento, en iberoamérica se han comunicado dos casos de este síndromed, 5 .

1. Servicio de Neuropsiquiatría Infantil, Hospital Clínico San Borja Arriarán. SSMC.

2. Departamento de Pediatría y Cingía Infantil, Facultad de Medicina, Campos Central, Universidad de Chile.

3. Unidad de Genética y Enfermedades Metabólicas, Instituto de Nutrición y Tecnología de los Alimentos, Utivcrsidad de Chile.

4. Departamento de Neuro-Oftalnología, Instituto de investigaciones Cerebrales Dc. Alfonso Asenjo. SSMO.

\section{Casos clínicos}

1. Lactante de sexo femenino, nacida del segundo embarazo nomal de padres jóvenes no consanguíneos. Un hermano sano. Parto de término, evtócico, Al nacer pesó $3600 \mathrm{~g}$, talla de nacimiento $50 \mathrm{~cm}$, perínetro craneano 35 $\mathrm{cm}$, Apgar 9 en uno y cinco min. Estuvo bien hasta la edad de dos y medio meses de vida en que se iniciaron crisis parciales que secundariamente se extendían a todo un hemicuerpo y cran seguidas de salvas de espasmos masivos flexores que no cesabon con fenobarbital, ácido valproico, piridoxina y prednisona. A la edad de cinco meses presentaba retraso severo de todas las áreas del desanollo psicomotor. sin dismorfias; perímetro craneano nomal; hipolonía axial; hiperreflexia y frecuentes salvas de espasmos en flexión. En el clectroencefalograma se registraba un patrón muy anormal de estallitto y supresión, dc mayor expresión en hemisferio izquicrdo. En la tomografía axial computadorizada (TAC) de cerebro se demostró agenesia parcial posterior del cuerpo calloso y un quiste aracnoidal cn la cistema pericallosa posterior. La radiografía de la columna vertebral mostró fusión parcial de las piczas D7 - D8. El rastreo metabólico, los aminoácidos en orina, sangre y líquido cerebro espinal, lactacidemia, amonemia y búsqueda de infecciones congénitas dieron resultados nomales. La evaluación papila óptica era de aspecto normal y se observaban placas de alrofia coriorretinal que recordaban lesiones de toxoplasmosis congénita.

Fue tratada con ACTH sintético $0,5 \mathrm{mg}$ - dosis i.m. tres veces por semana durante un mes, sin obtener remisión de crisis. Luego se administró una as ociación de ácido valproico y clonazepam, con lo cral se logró controlar parcialmenie los espasmos. En el úlitimo control, realizado al afto de edad, tenía severo daño neurológico, microcefalia, conısol елcefálico parcial, pobre contacto con el medio, visiôn dudosa, y sufría reiterados episodios de infección respiratoria. 
2. Niña nacida del primer embarazo de padres jóvenes no consanguireos. Embarazo sin problemas excepto por placenta de inserción baja. Ĺna ultrasonografía obstétrica hizo sospechar, al quinto mes de gestación, la posibilidad de hidrocefalia. Parto a las 38 semanas de gestación. Fórceps por expulsivo prolongado. Peso $3290 \mathrm{~g}$, talla $49 \mathrm{~cm}$ y perímetro crancano $34,5 \mathrm{~cm}$ al nacer. Apgar 8 y 9 uno y cinco minulos después. A los dos días de vida la taltraso. nografía registraba agenesía total de cuepo calloso asociada a dos quistes de la línea media cerebral. A la edad de 25 dias presentó crisis tónico-clónicas generalizadas y luego se agregaron salvas de espasmos en flexión y crisis parciales. En el electroencefalograma había actividad de fondo desorganizada de alto voltaje sobre la cual había un patrón hemilheta altemante en el lado izquierdo. No se encontraron dismorfias, salvo por escoliosis dorsal, ni alteraciones craneofaciales, tenía hipotonia muscular, sufría permanentes episodios parciales, consistentes en movimientos oculares y de manos, y espasmos en flexión. Las radiografías de la columna mostraban vértebras en mariposa y hemivértebra en T3. No se encontraron alteraciones metabólicas ni evidencia de infecciones congénitas. En los ojos había áreas de atrofia corioretinal bilaterales sin signos de actividad y coloboma del nervio óptico izquierdo.

Los episodios de espasmos masivos cedieron Iransitoriamente con la administración i.m. de ACTH sintécico por dos semanas, pero reaparecieron posterionmente sin responder a una segunda cura con ACTH. A pesar de la administración de ácido valproico, clobazam, carbamazepina, la paciente evolucionó con frecuentes crisis parciales securdariamente generalizadas y espasmos masivos, grave retardo del desartollo, síndrome piramidal bilateral y bronconeumonias repetióas, falleciendo a causa de una de éstas a la edad de un año.

\section{Comentario}

Estas pacientes cumplen con los tres requisitos básicos para el diagnóstico del síndrome de Aicardi. Los espasmos masivos son una de las características que definen esta condición. Esta forma de epilepsia del lactante se ha descrito en varias enfermedades neurológicas, entre ellas esclerosis tuberosa, fenilquetonuria, síndrome de Down, secuclas de prematuridad, infecciones del sistema nervioso central, de modo que siempre es necesaria una cxhaustiva investigación etiologica para identificar la condición de base. En pacientes con síndrome de Aicardi la manifestación epiléptica tiene algunas características que pueden orientar al diagnóstico correcto, entre ellas la aparición precoz de espasmos, generalmente antes de la edad de tres meses, los que a menudo se asocian a crisis parciales como en las niñas que se comentan. Con frecuencia los espasmos son asimétricos, es decir, comprometen en mayor medida a un hemicuerpo, fenómeno relativamente infrecuente en casos de otra etiología ${ }^{6}$. Se han descrito alteraciones características en el electroencefalograma de los pacientes con sindrome de Aicardi, precozmente en el curso de la enfermedad, en particular durante la vigilia, consistentes en un patrón de estallidosupresión, con completa asincronía entre ambos hemisferios, también registradas en las dos niñas de esta experiencia. Las alteraciones descritas disminuyen y se organizan durante el sueño. El patrón observado al comienzo de la enferme. dad suele evolucionar posteriormente a un trazado multifocal sobre un fondo severamente desorganizado $^{7,8}$, por lo que el electroencefalograma con estas características sería útil en el diagnóstico del síndrome.

Las anomalías del cuerpo calloso, agenesia parcial y total, en relación a quistes de la línea media, se han descrito en todos los casos comunicados, además de otras malformaciones como polimicrogiria. La agenesia del cuerpo calloso es una malformación del sistema nervioso central que la mayoría de las veces forma parte de una alteración más general que también afecta a otros sistemas. Se ha descrito en anomalías cromosómicas. ${ }^{10}$ y con herencia ligada al cromosoma $\mathrm{X}^{11}$, recesiva $^{12}$ y dominante ${ }^{13}$. De particular interés resulta la asociación de algunos enores innatos del metabolismo (hiperglicinemia no cetósica, acidosis láctica congénita, sindrome de Zellweger, deficiencia de piruvato carboxilasa y deficiencia de piruvato deshidrogenasa) con anomalías del cuerpo calloso, puesto que en estos casos ocurren con frecuencia convulsiones precoces de difícil tratamiento y alteraciones neurológicas seve$\operatorname{ras}^{14,15}$, de donde proviene la necesidad de descartar enfermedades metabólicas.

Las anomalías del fondo de ojo tienen gran valor en el diagnóstico del síndrome de Aicardi. En particular las lagunas coriorretinales constiluyen el signo más característico y se encuentran desde el nacimiento. Su hallazgo permitiría predecir la agenesia del cuerpo calloso. Se trata de áreas de hipopigmentación redondeadas, de ta. maño variable y borde pigmentado, bajo las cuales se visualizan vasos coroidales intactos ${ }^{16}$. Estudios histopatológicos han demostrado anormalidades del epitelio pigmentado de la retina, con proliferaciones tubulares y papilares alrededor del nervio 6 ptico $^{17}$. Las lesiones coriorretinales son semejantes a las secundarias a toxoplasmosis congénita, razón por la cual se las ha denominado "pseudotoxoplasmosis". 
La manifestación de este síndrome, exclusivamente en nifias, sugiere una herencia dominante ligada al cromosoma $X$, letal en varones. Sin embargo en 186 casos, en que 79 tenian 149 hermanas, no se encontraron hermanos o parientes afectados ${ }^{3}$ y sólo se ha comunicado un caso de sindrome de Aicardi en dos hermanas ${ }^{18}$. Los estudios con sondas de mapeo de $\mathrm{DNA}^{19}$ y de inactivación de cromosoma $\mathrm{X}^{20}$, no han dado resultados positivos, lo que sugiere que las alteraciones moleculares serían heterogéneas y que se trataría de nuevas mutaciones, por lo que el consejo genético debe ser muy cauteloso en estos casos.

\section{Resumen}

Se describen dos niñas con crisis epilépticas en los primeros meses de vida, que evolucionaron a espasmos infantiles; agenesia de cuerpo calloso; coriorretinopatía caraclerizada por atrofia lacunar; severo retardo del desarrollo psicomotor y malformaciones vertebrales, asociación que corresponde al síndrome de Aicardi, de herencia dominante ligado al cromosoma $X$ y probablemente producido por una mutación reciente. Puesto que los criterios mayores que definen el síndrome pueden verse también, cuando aparecen por separado, en otras afecciones genéticas o metabólicas, es necesario efectuar un cuidadoso diagnóstico diferencial.

(Palabras clave: agenesia del cuerpo calloso, espasmos infantiles, coriorretinopatía atrófica lacunar, sindrome de Aicardi.)

\section{Agradecimientos}

A la Dra. Camen Gayewski por la derivación de una de las pacientes, a las Dras. Mana Colombo y Fanny Cortés por su aporte en la evaluación metabslica y genćuica de las pacientes.

\section{Referencias}

1. Aicardi J, Lefevre J, Lerique-Koechlin A: A nes syndrome: spams in flexion, callosal agenesis, ocular abnomalities. Electroencephalogr Clin Neurophisiol 1965; 19:609-610.

2. Aicardi $J$, Chevrie $J J$, Rousselie $F$ : Le syndrome spasmes en flexion, agenesie calleuse, anomalies chorioretiniennes. Arch Fr Pediatr 1969; 26: 1103. 1120.

3. Aicardi J, Chevrie JJ, Baraton $J:$ Agenesis of the corpus callosum. Hadbook of Clinical Neurology, Vol. $6(50)$ : Malformations. Ed. NC Myrianthopoulos. Elsevier Science Publisher B.V. 1987: 149-173.

4. Base de datos LILACS, 1982-1991. Sistema Nacional de Información en Ciencias de la Salud. Centro Latinoamericano y del Caribe de Información en Ciencias de la Salud.

5. Abraham $R$, Kando PA. Silva W. Mello P. Segre NG: Sindrome de Aicardi: relato de dos casos. Arq Neuropsiquiatt $1986 ; 44: 364-372$.

6. Hrachovy RA, Frost $J D$ : Intensive monitoring of infantile spasms. En: Intractable Epilepsy. Eds. D. Schimidt y PL Morselli. Raven Press, NY, 1986: 147-156.

7. Jeavons PM. Green Sh, Oldhom JS: The EEG diagnosis of Aicardi syndrome. Electroencephalogr Clin Neurophysiol $1973 ; 35 \div 422$.

8. Fariello $R$, Chun $R$, Doro $J$ es al:: EEG recognilion of Aicardi's syndrome. Arch Neurol 1977; 34: 563-566.

9. Zellweger $H$, Ionasescu V, Schochet $S$, et al.: Chromosomal aneuploidies excluding Down syndrome. En: Handbook of Clinical Neurology vol. 31. Eds. PJ Vinken and GW Bruyn. Congenital Malformations of the Brain and Skull, Part II. Amsterdarn, North-Holland Publ. Co. 1977: 471-547.

10. Sumi SM: Brain malformations in the trisomy 18 syndrome. Brain 1970; 93: 821-830.

11. Menkes JH, Philippart $M, C$ lark $D B$ : Hereditary partial agenesis of corpus callosum. Arch Neurol 1964; 11 : 198-208.

12. Kaplan $P$ : X-linked recessive inheritance of agenesis of the comus callosum. Am J Med Genet 1983; 20: $122 \cdot 124$.

13. Lynn RB, Buchanan DC, Fenichel GM, es al.: Agenesis of the corpus callosum. Arch Neurol 1980; 37: 444 . 445.

14. Banforth F, Bamforth S, Poskit $K$, at al.: Abnornalities of corpus callosum in patients with inherited metabolic diseases. Lancet 1988; 2: 451.

15. Dobyns WB: Agenesis of the corpus callosum and gyral malfomations are frequent manifestations of nonkelotic hyperglycemia. Neurology 1989; 39; 817.820 .

16. Hoyt CS, Billson F, Ouvner R, et al.: Ocular features of Aicardi's syndrome. Arch Ophtalmol 1978; $96: 291$ 295.

17. Foni RL, Marines HM, Carlwright $J$, el al.: Aicardi syndrome: A clinicopathologic case repon including electron microscopic observations. Ophsaimology 1991; 98: 1727-1731.

18. Moina JA, Mateos F, Merino M, el al.: Aicardi syndrome in two sisters. J Pediatr 1989; 115: 282-283.

19. Brondum Nielsen $K$, Anvret $M$. Flodmark $O$, et at.: Aicardi syndrome: Early neuroradiologicat manifes. tations and results of DNA studies in one patient. Am J Med Geret 1991; 38: 65-68.

20. Neidich JA, Nusbowm RI, Packer RJ, et al: Heterogeneily of clinical severity and molecular lesions in Aicardi syndrome. J Pediatr 1990: 116: $911-917$. 